\title{
Analysis and Design of Independent Pitch Control System
}

\author{
CHU Yun Kai ${ }^{1, a^{*},}$ MIAO Qiang ${ }^{2, b}$, DU Jin Song ${ }^{1, c}$, LIU Yi Yang ${ }^{1, d}$ and SU Rui ${ }^{1, e}$ \\ ${ }^{1}$ Shenyang Institute of Automation Chinese Academy of Sciences, Shenyang, 110016, China \\ ${ }^{2}$ Chengdu Forward Technology Co.Ltd, Chengdu, 610000, China \\ achuyunkai@sia.cn, ${ }^{b}$ michael@sc-forward.com, ${ }^{c} j s d u @ s i a . c n,{ }^{d}$ sialiuyiyang@sia.cn, ${ }^{e}$ surui@sia.cn
}

Keywords: Pitch control system; Pitch controller; Backup power

Abstract: As the most important part of wind turbine, the safety and reliability of pitch control system is very important. This paper deeply studies three aspects of pitch control system, including pitch controller, servo driver and backup power. At the basis of the control principle of pitch control system, the overall design content of the system is presented. Furthermore, the system has been successfully applied in engineering practice. The experimental results show that the system has a good response speed and control precision, and fully meets the technological requirements of pitch control system.

\section{Introduction}

In recent years, with the growing concern of the environmental and energy supply issues, the wind power technology has been rapid developed [1],[2],[3]. As the most important part of wind turbine, pitch control system has been continuously in-depth studied, and its functions are more and more complex which lead to the numbers of control devices and sensors are greatly increased[4],[5]. So in the condition of hub space is very narrow and the quantity and volume of electrical components are limited, how to implement all functions and stable control are the urgent problems to be solved [6].

In this paper, each blade has an independent pitch controller which was developed independently based on DSP2812. The pitch controller connect main control unit, pitch drive unit and redundant encoder by the way of CAN bus, and effective integrate the functions of backup power manage and pitch load analyses, so the structure of pitch system is more optimized and the operation is more flexible. The new pitch system has the characteristics of high integration, high maintainability and low failure rate. All the controll functions of the system had been tested on the $1.5 \mathrm{MW}$ wind turbine.

\section{Pitch Control System Overall Design}

At present, the domestic pitch control system widely uses the independent control strategy[7]. According to function, the pitch system can be divided to three main parts: pitch controller, pitch driver and backup power. The overall design scheme is shown in Fig.1. 


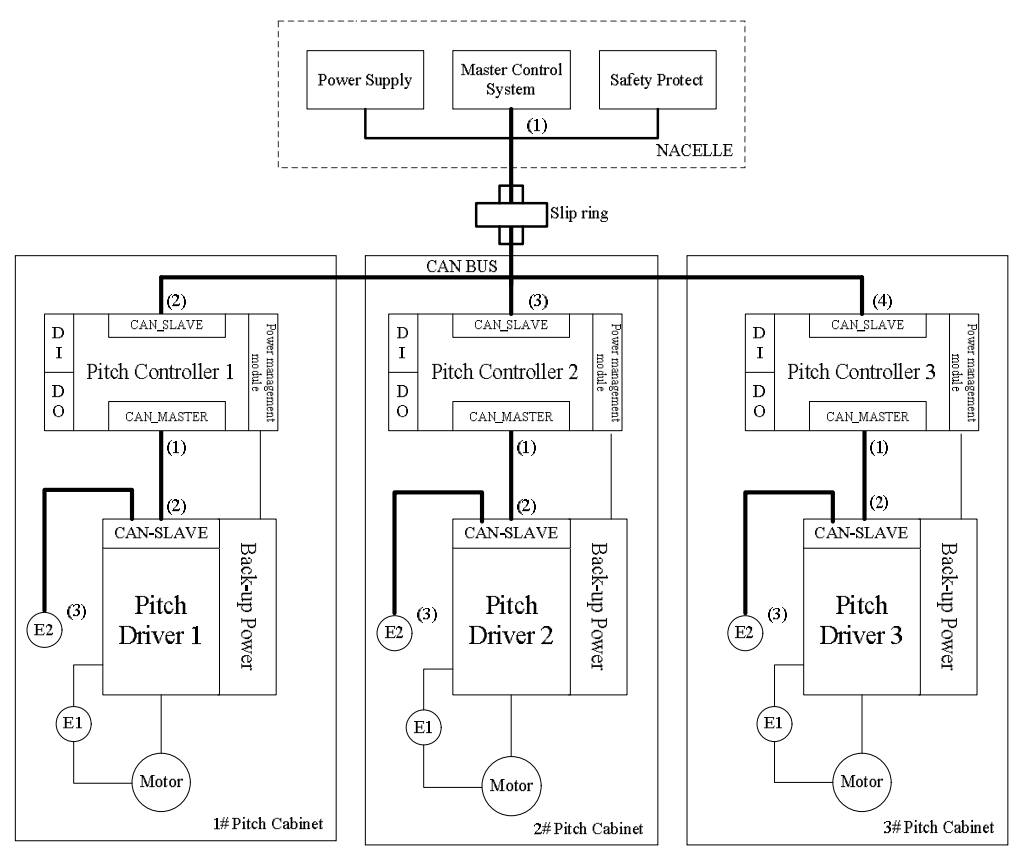

Fig.1. Pitch system overall design scheme

The whole system adopts CAN bus communication and uses slip ring to connect the power and communication between pitch system and master control system. Three independent pitch controllers receive control command and fault diagnosis from master controller respectively, and send back their operating mode. The pitch controller will analyze the pitch load and add the calculated value to the blade angle and speed from master controller, and transmit the final value to the corresponding drive unit to realize the independent pitch function. At the same time, pitch controller collects real-time values of blade angle both from pitch driver and redundant encoder E2, if the two collected values are not the same, system will go into emergency closing pitch mode. When the slip ring power supply disconnect, pitch controller will adopts the back-up power to close the pitch.

\section{Pitch Controller Design}

The pitch controller is designed by TI company TMS320F2812 microcontroller as the control core, extension modules contain DI, DO, AI, CAN-master, CAN-slave, power management and so on. The single pitch system structure diagram is shown in Fig.2.

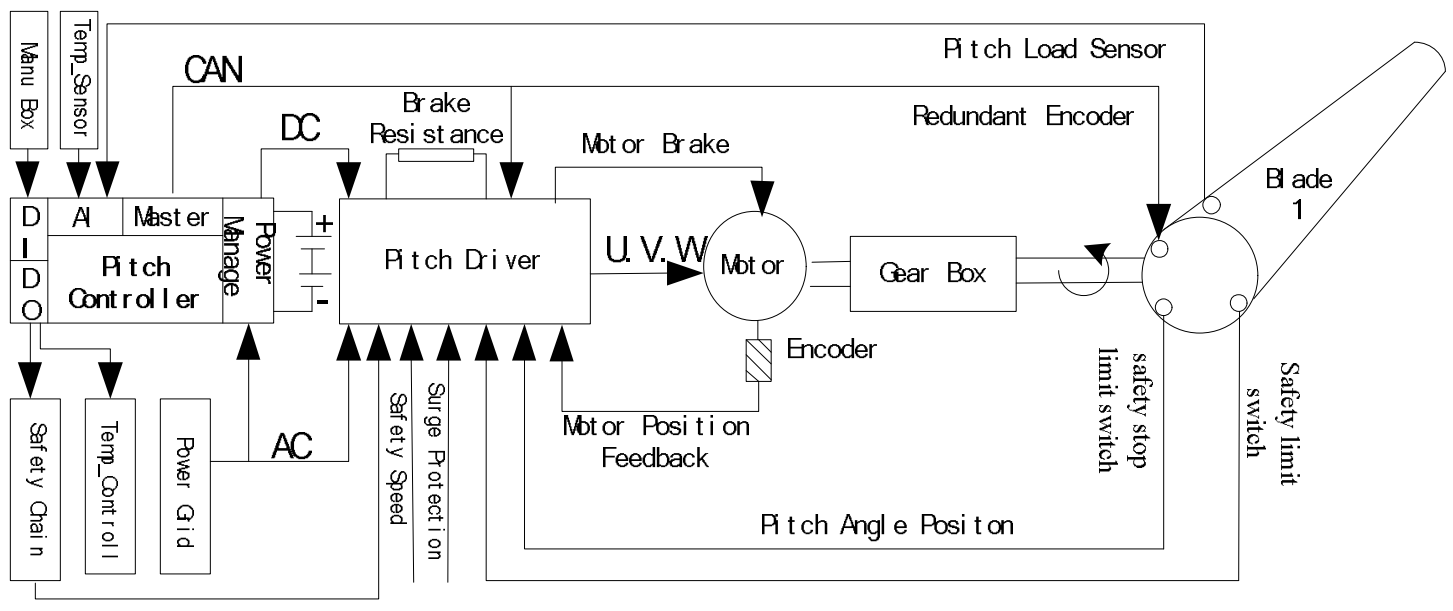

Fig.2. Single pitch system structure diagram

DI module is used to connect manual control box for the system's maintenance. DO module is used to control the safety chain and the temperature of hub. AI module is used to collect blade load values from the sensor which installed on blade root for the decoupling control. CAN modules are used to link pitch driver and redundent encoder. Power management module used for the back-up power's charge, 
discharge and switch the supply power. Safety stop limit switch and safety limit switch are installed on the blade gear and connect to the pitch driver directly, when any switch is triggered, pitch controller will disconnect the safety chain and other two blades will be emergency closing.

\section{Pitch Driver Selection}

The torque value of blade root is different when pitch control system in different operating mode. In normal operating mode, pitch speed is about $4^{\circ} / s$, and in emergency closing pitch mode, the torque requirement is relatively higher, pitch speed will reach $8^{\circ} / s$, so the pitch driver selection in this paper considers the torque requirement in emergency closing pitch mode. According to the $1.5 \mathrm{MW}$ wind turbine technical requirements, in the condition of wind speed is $20 \mathrm{~m} / \mathrm{s}$ and emergency closing pitch speed is $8^{\circ} / \mathrm{s}$, the torque of drive motor $T \geq 25 \mathrm{~N} \bullet \mathrm{m}$. The gear ratio between blade and motor is 1500 , so the rated speed of motor is as follows:

$$
n_{N}=\left(\frac{8}{360} \times 60 \times 1500\right) r / \mathrm{min}=2000 \mathrm{r} / \mathrm{min}
$$

The motor angular velocity $\omega$ is

$$
\omega=\frac{8 \times 1500 \times 2 \pi}{360}=66.7 \pi=209.4 / s
$$

According to motor torque, power and angular velocity, the motor rated power can conclude as follows:

$$
P_{N}=T_{N} \times \omega=25 \times 209.4=5.23 \mathrm{~kW}
$$

So this system selects the rated speed $n_{N}=2000 \mathrm{r} / \mathrm{min}$, rated torque $T_{N}=25 N \bullet \mathrm{m}$ and maximum torque $T_{M} \geq 33.7 \mathrm{~N} \bullet m$ (considering the coefficient of safety allowance 1.35) motor as the pitch drive unit.

According to the hub space where pitch motor located is narrow and ensure the motor to long time normal operation in the harsh and complex environment, the system choose the permanent magnet synchronous motor (PMSM) as the drive unit, PMSM has lots of advantages, such as high efficiency, high energy density, high torque inertia ratio. For the system design requirements, pitch driver need to configure the CAN communication interface card and it could be powered by $300 \mathrm{~V}$ DC through the DC bus terminal. Considering technology parameters and function requirements, the system chooses the SEW brand MDX61B 5 a3-0075-4-0 t model drive controller and CFM 112 s/BR/TF/AS1H/SB50 model PMSM which the biggest dynamic torque can be reached $39.6 \mathrm{~N} \bullet \mathrm{m}$.

\section{Backup Power Management System Design}

At present, the back-up power system in pitch system widely use charging batteries which based on principle of electrochemical reaction, however, due to the problem of cycle life, high and low temperature performance, it will have limitation in application. With the continuous development of material science, supercapacitor is widely used in engineering projects, compare with other storage technology, it possessed the features of long cycle life, high specific power, fast charging and discharge rate and so on. This system adopt supercapacitor as the backup power supply, when the power drop from the slip ring, the power management module will switch supercapacitor to power pitch driver through the DC bus terminal, ensure the blade safety closed. The back-up power management structure is shown in Fig.3. 


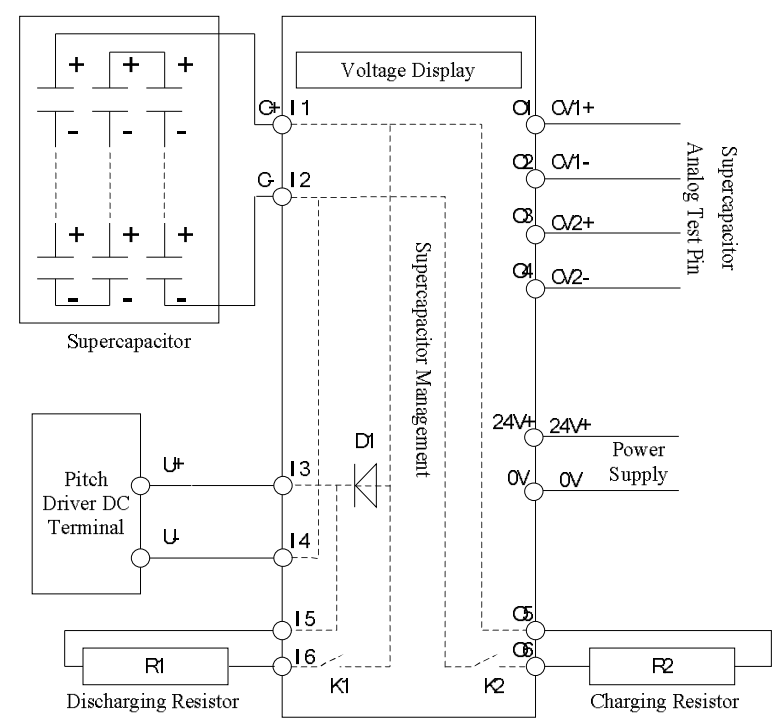

Fig.3. Back-up power management structure

Each blade control unit equipped with a set of supercapacitor, when the power supply from slip ring is normal, the management module will real-time monitor the supercapacitor's voltage, when the value is above or below the set value, controller will sends error message to master. The pin I1, I2 of power management module respectively connect to the pin $\mathrm{C}+, \mathrm{C}$ - of supercapacitor, pin $\mathrm{I} 3$, I4 connect to the DC input terminal L+, L- of pitch driver, pin I5,I6 connect charging resistor, pin O5, O6 connect discharging resistor. In the case of pitch system in normal operation mode, if the DC terminal voltage of pitch driver is higher than supercapacitor, controller will close contactor $\mathrm{K} 1$ to charge supercapacitor through charging resistor. Conversely when the DC terminal voltage of pitch driver is lower than supercapacitor, supercapacitor will provide power to pitch driver through diode D1 automatically. When the system needs to empty the energy of back-up power, controller will close contactor $\mathrm{K} 2$ to discharge through resistor $\mathrm{R} 2$ until the process finish. Pin $\mathrm{O} 1, \mathrm{O} 2$ are the full voltage analog output of supercapacitor and pin $\mathrm{O} 3, \mathrm{O} 4$ are the semi-voltage analog output, the output ratio is 0-500VDC corresponding 0-5VDC, used for external measure.

\section{Blade Load Analysis Design}

During the operation of wind turbine, blade load change with the rotation of hub and change of pitch angle and wind speed. For example when the blade in the zero open state and rotate to the vertical above position of hub rotating horizontal, the blade load is the maximum value, similarly, when the blade in the close state and rotate to the vertical below position of hub rotating horizontal, the blade load is the minimum value, and the wind speed at the top and bottom of hub rotating horizontal is also different, all these factors will lead to wind turbine vibrate, affect output power and reduce the service life. Base on the above analysis, add the blade load calculation unit in the pitch controller. The blade load analysis design principle diagram is shown in Fig.4.

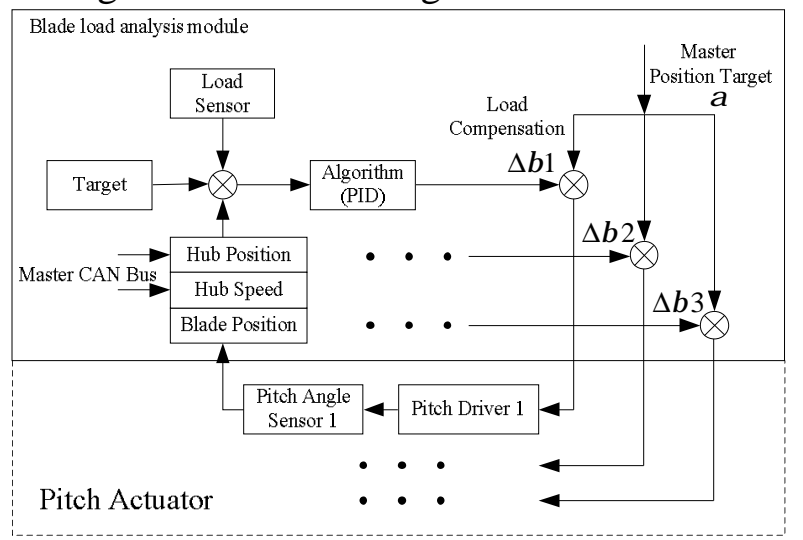

Fig.4. Blade load analysis principle diagram 
The analysis unit uses the value from master controller as the target and value form the load sensor as feedback to construct a PID closed-loop control, and base on the hub position, hub rotate speed and blade angle from redundant encoder to calculate the angle compensation for each blade $\Delta \beta 1, \Delta \beta 2$ 、 $\Delta \beta 3$, add the value to the target blade angle from master controller and send the added values $\alpha+\Delta \beta 1, \alpha+\Delta \beta 2, \alpha+\Delta \beta 3$ to the corresponding drive unit to complete the blade load compensation. Through this function to ensure the force balance of the wind turbine, reduce the vibration in the operation process and improve the overall performance.

\section{Experimental Results and Analysis}

This pitch control system use the $1.5 \mathrm{MW}$ experimental wind turbine as the platform for the system performance test. When the wind speed is $2 \mathrm{~m} / \mathrm{s}$, pitch system control the blade continuous open and close two times in the speed of $6^{\circ} / s$ and emergency pitch close in the speed of $8^{\circ} / s$ at last. The pitch control system test curve is shown in Fig.5.

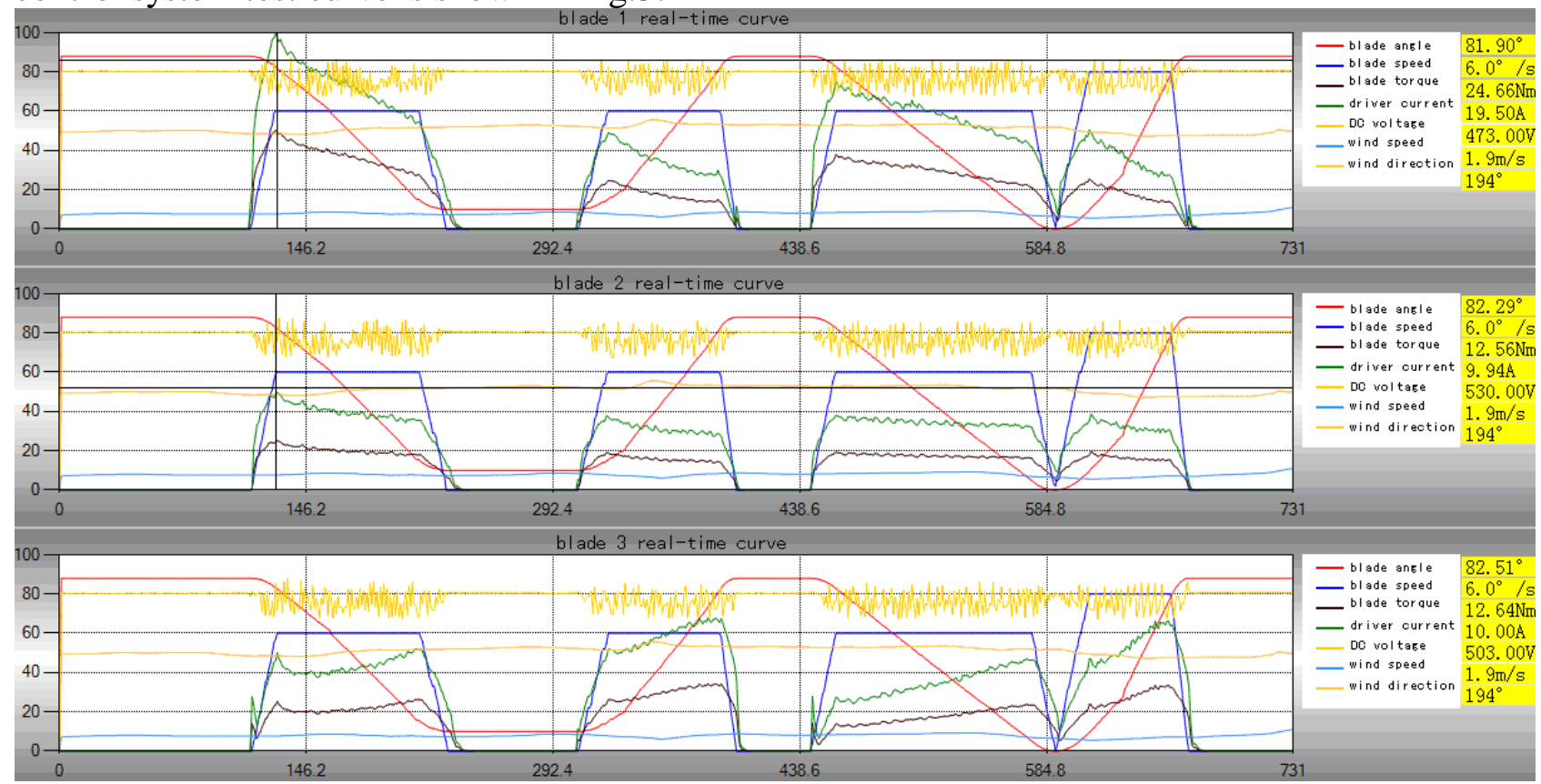

Fig.5. Pitch control system test curve

From the testing curve: Due to the 1\# blade is in the vertical direction, the maximum torque value is $24.66 \mathrm{Nm}$ and the maximum current value is $19.5 \mathrm{~A}$. For the other two blades, the maximum torque value is $12.64 \mathrm{Nm}$ and the maximum current value is $9.94 \mathrm{~A}$. For the maximum torque and current of the driver is $39.6 \mathrm{Nm}$ and $35 \mathrm{~A}$, the pitch driver module is fully meet the requirement of the wind turbine. From the curve of blade speed, blade angle and emergency pitch close process, the pitch control system has well response and control precision, and three blades can maintain good consistency.

When the wind speed is $8 \mathrm{~m} / \mathrm{s}$, the pitch controller makes the back-up power test, uses the supercapacitor to open and close blade continuously until the back-up power stop work. The supercapacitor test curve is shown in Fig.6. 


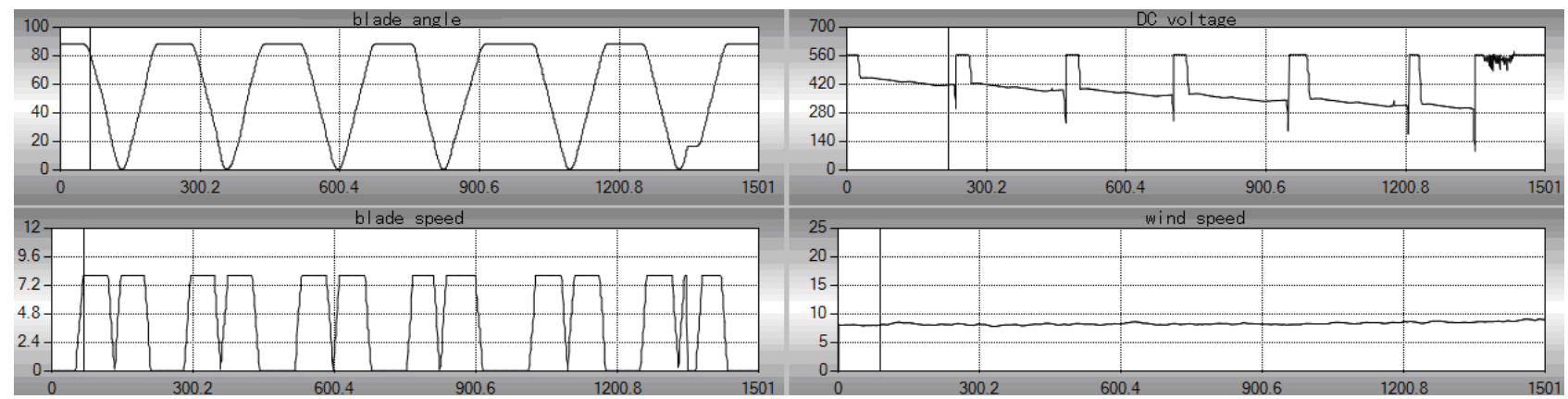

Fig.6. Supercapacitor test curve

From the test curve: Using the backup power supply, pitch system can continue open and close blade five times at least. When the voltage of supercapacitor down to 300VDC, pitch controller switches the power supply from slip ring, it fully meets the requirement of wind turbine for backup power system. At the same time, the system sets the fault back-up power voltage to $360 \mathrm{~V}$ and warning back-up power voltage to $420 \mathrm{~V}$.

\section{Conclusion}

In this paper, we use the independent pitch controller, SEW servo drive system, supercapacitor as the backup power and CAN bus communication to realize the pitch control system, so, the whole control system has structure simplify and high reliability. The experimental results have showed that the system has highly response speed and control precision and it can meet the requirement of pitch system for MW rated wind turbine. The system will have a very wide application prospect.

\section{Acknowledgement}

This work is partially supported by the Funds of National Science of China (Grant No. 61203329).

\section{References}

[1] Paul G Leahy; Antonino Marvuglia; Eamon J McKeogh,. Current methods and advances in forecasting of wind power generation Foley, Aoife M.; Renewable Energy, 2012, v 37, n 1, p 1-8.

[2] Uski Sanna; Kim Inna. Assessment of wind power impact on power system transmission losses. IEEE PES Innovative Smart Grid Technologies Conference Europe, 2014, p1659-1663.

[3] Dapeng Chai; Hui Shi; Song Zhe. Simulation of deterministic model and stochastic model of additional cost for grid-integration of wind power. Power System Technology, 2014 , v 38, n 3, p 687-693.

[4] Haijun Ren; Yulin He. Comparative analysis on two single neuron PID control methods for wind turbine pitch-control system. Power System Technology, 2010, v 34, n 12, p 154-157.

[5] Xiuxing Yin;Yonggang Li. Integrated pitch control for wind turbine based on a novel pitch control system. Journal of Renewable and Sustainable Energy, 2014, v 6, n 4, p123-129

[6] Shi Yin; Zhongyuan Yu; Kaifeng Meng. Fault identification of pitch control system of wind turbine based on nonlinear state estimation. Proceedings of the Chinese Society of Electrical Engineering, 2014 , v 34, p 160-165.

[7] Xianzheng Liu; Xingcheng Wang; kai Zheng; Jinyuan Li. Design of variable pitch-control system based on two-degree-of-freedom internal model control. 2014 International Conference on Information Science, Electronics and Electrical Engineering, 2014, v 2, p 1348-1352. 